\title{
Rehabilitation to Improve the Function and Quality of Life of Soft Tissue and Bony Sarcoma Patients
}

This article was published in the following Dove Press journal:

Patient Related Outcome Measures

\author{
Cody C Andrews' \\ Geoffrey Siegel ${ }^{2}$ \\ Sean Smith (1D) \\ 'Department of Physical Medicine and \\ Rehabilitation, University of Michigan, \\ Ann Arbor, MI, USA; ${ }^{2}$ Department of \\ Orthopaedic Surgery, University of \\ Michigan, Ann Arbor, MI, USA
}

\begin{abstract}
Sarcomas are bone and soft tissue tumors that can have significant effects on patient function and quality of life. Like most malignancies, treatment includes a combination of chemotherapy, radiation, and surgical resection, all of which also carry risks and long-term effects. A multidisciplinary rehabilitation plan can help minimize symptoms and sequelae which negatively affect the patient function and quality of life, including pain, chemotherapy-induced peripheral neuropathy, radiation fibrosis, activity restrictions following surgical excision, amputation, bowel and bladder dysfunction, and lymphedema. Patients should be evaluated by a rehabilitation specialist at any point during their diagnosis, treatment, and survivorship phase to determine appropriate interventions to minimize the impact of sarcomas and their treatment on patient function and quality of life.
\end{abstract}

Keywords: soft tissue sarcoma, bone sarcoma, rehabilitation, sarcoma rehabilitation, function, quality of life, limb salvage, amputation

\section{Introduction}

Sarcomas are rare malignancies arising from mesenchymal cells which can affect bone, muscle, adipose tissue, cartilage, and the peripheral nervous system and have wideranging deleterious effects on quality of life (QOL) and function based on tumor pathology, location, and treatment. ${ }^{1-4}$ Sarcomas may occur anywhere in the body and most commonly metastasize to the lungs. Overall mortality is high, with roughly half of the patients eventually dying from the disease. ${ }^{5}$ Like most cancers, treatment consists of a combination of surgical excision, chemotherapy, and/or radiation. Given the wide variety of disease presentation and varied treatment options available, each patient will face unique challenges that are best addressed by a multidisciplinary team. ${ }^{6}$

Cancer survivorship and patient quality of life are an increasingly important part of oncologic treatment. As patients survive longer and cure rates increase, more attention is being paid to the long-term experience of these patients. ${ }^{7}$ Poor physical function from sarcoma and its treatment has been tied to decreased QOL and a lower rate of returning to work. ${ }^{8-10}$ Pain, fatigue, cognitive impairment, anxiety and depression, and impaired social participation are also common. ${ }^{11}$ The management of these patients and their symptoms should be rooted in an understanding of the treatments patients undergo and management options available for symptoms they experience.

\section{Surgical Management}

The role of surgery in the realm of bone and soft tissue sarcomas (STS) has transitioned from an ablative option to a focus on limb salvage in the past 40
Correspondence: Cody C Andrews Department of Physical Medicine and Rehabilitation, University of Michigan, 325 E. Eisenhower Pkwy, Ann Arbor, MI 48108, USA

Tel $+1734-936-7175$

Fax +| 734-615-67|3

Email coan@med.umich.edu 
years. Every bone sarcoma and STS is different and every surgery is tailored specifically to the patient, and functional expectations should be planned according to the soft tissue and bone resected. Expected loss of function should be discussed with the patient pre-operatively, though oftentimes involvement of neurovascular structures cannot be elucidated until the time of surgery. ${ }^{12,13}$ It is sometimes also necessary to plan for complex plastic surgery reconstruction of soft tissue defects, which also has an implication in ultimate function, and may necessitate range of motion restrictions in the immediate postoperative period. ${ }^{14}$ The surgeon's goal should be first and foremost wide resection of the sarcoma. ${ }^{15,16}$

Limb salvage surgery is possible due to the availability of both allograft and off-the-shelf modular endoprosthetic reconstructions. Orthopedic surgeons have the capability to reconstruct the entire femur, tibia and humerus with the surgeon's preference of a combination of these constructs. The functional goals at the time of surgery are to recreate the patient's normal limb length and alignment and to give them a painless joint with functional range of motion. ${ }^{17}$ There are many nuances and preferences with respect to surgical technique, and very few randomized prospective studies exist highlighting the benefit of one technique over another. Those patients who survive long enough may have to undergo revision surgery/surgeries due to many factors including wear, failure, and infection. ${ }^{18}$ Potential contraindications to limb salvage include advanced patient age, immature skeletal age, extensive disease or disease unlikely to respond to chemotherapy and/or radiation, distal extremity disease, and extensive involvement of neurovascular structures. ${ }^{19}$

\section{Lower Extremity Sarcomas}

Femoral sarcomas are split into three categories: proximal, diaphyseal, and distal, and reconstructions can consist of any combination of those three. Proximal tumors usually require reconstruction of the femoral head, either as a hip hemiarthroplasty or total hip arthroplasty. It is the surgeon's responsibility to provide a stable hip joint, but often soft tissue constraints of the hip are compromised due to the necessity of wide resection of the tumor. Rehabilitation goals should focus on diminishing the chance for hip dislocation postoperatively and strengthening surrounding muscles. ${ }^{20}$ Depending on the tumor burden and available bony architecture after resection, the decision may be made for resection arthroplasty or "girdlestone" procedure where the proximal femur is not reconstructed after resection. This does have a significant impact on postoperative rehabilitation and definitive function, but many of these patients are ultimately able to ambulate at a modified-independent level. ${ }^{21}$ Sarcomas requiring resection of femoral diaphyseal bone but sparing the hip and knee joints can be reconstructed with a variety of allograft and metal component options with high functional expectations. ${ }^{22}$ Distal femoral resections and reconstructions are often technically demanding due to the close proximity of many anatomic structures including popliteal artery and vein as well as tibial and common peroneal nerves. The knee itself is a superficial joint without a deep soft-tissue envelope and therefore prone to wound complications and prosthetic joint infections. Nevertheless, limb salvage surgery is usually successful, but patients should be properly counselled and should prepare for aggressive post-surgical rehabilitation in an effort to prevent arthrofibrosis. ${ }^{23}$

Tibial resections and reconstructions have a much more unpredictable result and are prone to a higher complication rate. Proximal tibial resections require complex reconstruction of the extensor mechanism of the knee which is prone to failure. Distal tibial resections require reconstruction of the ankle joint, which is an inherently unstable joint requiring complex soft tissue reconstruction for stability, which is again prone to failure. These patients should be prepared for a prolonged treatment and rehabilitation course. ${ }^{24}$

The decision between limb-sparing surgery and amputation is ultimately highly personal and depends on the goals of the patient when a choice is medically feasible. Overall, rates of amputation for sarcoma have dropped significantly in recent decades in favor of limb salvage surgery. ${ }^{25}$ Most studies show no difference in QOL between patients who undergo amputation and those who undergo limb-sparing surgery, though each intervention offers different potential levels of function. ${ }^{19}$ Aksnes et al showed significantly worse clinician-reported, but not patient-reported, functioning for osteosarcoma patients who underwent amputation. ${ }^{25}$ For patients with tumors distal to the knee, amputation and limb-salvage seem to be equivalent in functional outcomes. ${ }^{25,26}$

Limb salvage is, however, not without drawbacks in terms of lower extremity use even if patients report having a similar QOL when compared to patients who undergo amputation. Quadriceps activation has been shown to be altered, ${ }^{27}$ and patients over one year out of limb salvage surgery have a high risk of impaired activities involving 
bending their knees, with associated decreased range of motion. ${ }^{28}$ Finally, patients may also prefer limb-sparing surgery for cosmetic reasons, which should be taken into consideration when planning a surgery.

One of the most important considerations is the desired level of activity for the patient, and for patients hoping to participate in higher-level physical activities, amputation may be desirable compared to limb-salvage surgery. Amputation followed by prosthetic restoration allows for higher impact physical activity than limb salvage. ${ }^{2}$ Given that many sarcoma patients are young and active, amputation may provide an avenue to remain active in many sports, albeit with the necessary adaptive equipment, such as a sprinting blade for an amputee who wishes to run. Unsurprisingly, functional outcomes are better for below the knee than above the knee amputation. ${ }^{25,26,29}$

Rotationplasty is a rarely offered but specialized procedure generally reserved for younger patients with distal femoral or proximal tibial lesions in which the distal lower extremity is rotated 180 degrees and attached the proximal femur, effectively replacing the knee with the ankle. Patients can be fitted with a specialized prosthesis when healing has completed. Due to the longer lever arm and functional joint, energy expenditure is less with ambulation. ${ }^{3}$ Patient QOL and function are at least equivalent to amputation or traditional limb salvage procedures. In one study, rotationplasty patients over the age of 24 even had higher physical and psychological well-being than younger patients. ${ }^{30}$ Abnormal cosmesis is a primary concern for many patients. ${ }^{2}$

\section{Upper Extremity Sarcomas}

Like the femur, humeral sarcoma resections and reconstructions are also split into any combination of three categories: proximal, diaphyseal, and distal. The realistic functional goal of humeral reconstructions is to give the patient a stable pedestal to allow for full use of the hand. Most often these patients lose significant shoulder function and should not expect over-head motion or a fully functioning rotator cuff. ${ }^{31}$ Given the importance of the hand and the crudeness of even the most advanced myoelectric upper extremity prostheses, limb-salvage is preferred in all situations when not medically contraindicated.

\section{Pelvic Sarcomas}

Due to the nature of the anatomy, pelvic resections for sarcomas are complex, technically demanding and highly variable. In relation to the tumor, the surgeon must consider the locations of the femoral and sciatic nerves (as well as lumbar plexus), aorta, inferior vena cava, common, internal and external iliac arteries and veins, urogenital structures including the ureter, bladder, prostate, spermatic cord, base of penis, vaginal wall, as well as gastrointestinal structures such as the peritoneum, bowel, rectum and anus, on top of all of the muscular attachments to the bony pelvis. When it comes to limb preservation, the so-called internal hemipelvectomy is the most common procedure, and there is considerable controversy regarding reconstruction of the bony pelvis or leaving the limb flail. ${ }^{32-34}$ What is certain is that complication rates are high, rehabilitation is long, and quality of life and functional outcomes are significantly reduced after these surgeries. ${ }^{35}$

\section{Spine Sarcomas}

Sarcomas of the spine, including chordomas, are difficult to treat due to proximity to the spinal cord and exiting nerve roots. Chordomas are bony sarcomas which occur in the axial spine, most often in the sacrum. ${ }^{36-41}$ They tend to be indolent with low rates of metastasis, but due to their location can have profound effects on patient function and quality of life. Patients may experience sensory changes, weakness, neurogenic bowel and bladder, and sexual dysfunction. ${ }^{42,43}$ With any spine tumor, the foremost concern is spine stability and spinal cord integrity. Any new or progressive myelopathic symptoms require immediate imaging and surgical consultation for decompression and stabilization.

The complex anatomy of the spine makes surgical resection of sarcomas and reconstruction difficult. The surgical goal is still negative margin resection with preservation of neurologic function and palliation of pain. However, often these tumors involve multiple spinal segments and significant functional loss is weighed in the face of wide margin surgery. Patients should be counselled preoperatively regarding the expected loss of function based upon pre-operative imaging of the tumor. ${ }^{44}$

Myelopathy from spine sarcomas (and malignancy in general) tends to be incomplete, presenting with myotomal weakness and dermatomal sensory loss with or without neuropathic pain. Patients should be carefully evaluated for the need for orthoses and adaptive equipment to maximize mobility and preserve energy. Neurogenic bowel and bladder dysfunction are common sequelae of spine tumors and treatment. These are typically classified as upper motor neuron (UMN) or lower motor neuron (LMN) patterns. UMN patterns tend to occur with spinal cord compression cranial to the cauda equina. Involvement of the S3 nerve root, either from direct tumor compression or 
surgical sacrifice, has been shown to predict development of LMN patterns of dysfunction. ${ }^{37,45-47}$

UMN patterns tend to present with spasticity, while LMN patterns tend to present with flaccidity. Thorough physical examination should be performed in patients with abnormal or altered bowel and bladder patterns after spine sarcoma treatment. Presence of sacral reflexes is of particular importance. Voiding diaries may be helpful. Urology referral for urodynamic testing should be considered as well. Abdominal imaging and stool studies may be helpful. ${ }^{43}$

If spasticity is present which is painful or functionally limiting, treatment with oral agents (such as baclofen and tizanidine) should be considered. Local injection with botulinum toxin or phenol nerve ablation may be beneficial for patients who cannot tolerate or do not derive sufficient benefit from oral regimens. Stretching is always indicated to treat spastic muscles.

Patients with spastic UMN bowel and bladder are usually treated with anticholinergic agents such as oxybutynin. Intermittent self-catheterization (ISC) is often required. Long-term indwelling catheters should be avoided due to the increased risk of infection and bladder cancer. ${ }^{48}$ A bowel regimen consisting of softeners and stimulants should be titrated such that a patient has a complete bowel movement every day or every other day. Patients may be able to utilize sacral reflexes to achieve bowel continence using suppositories and/or digital stimulation to promote emptying.

Lower motor neuron patterns in which the sacral reflexes are weak or absent, which is the expected pattern in symptomatic sacral chordoma patients, tend to be more difficult to treat. Patients may benefit from cholinergic agents such as bethanechol to promote bowel and bladder contractility. Again, ISC is often required as patients are at risk for bladder distention and incomplete emptying. Bulking agents, such as fiber supplements, and manual stool removal are the mainstay of bowel management. Of note, digital stimulation and suppositories will be ineffective.

Patients who receive radiation to the sacrum are also at risk for radiation fibrosis of the muscles and nerves of the pelvic floor, which can exacerbate neurogenic bowel and bladder symptoms and be profoundly painful. Pelvic floor therapy should be strongly considered for these patients. Interventional procedures such as ganglion impar block may help control pain.

\section{Radiation and Chemotherapy}

Though surgery is the mainstay treatment of most sarcomas, many patients also receive chemotherapy and/or radiation, usually in an effort to shrink tumors before surgical excision or to control tumor burden after incomplete debulking. These treatments can also cause side effects which can decrease function and QOL. A thorough history of the treatments a patient has received in addition to surgery is important to obtain for proper evaluation of symptoms and dysfunction and for appropriate monitoring for late treatment effects.

Many chemotherapy agents (e.g. vincristine, platinumcontaining agents) can cause peripheral neuropathy. Chemotherapy, especially platinum-containing agents, is typically reserved for adjuvant treatment of bony sarcomas and its role is less defined in STS. In addition to neuropathic pain, the loss of sensation, proprioception, and distal muscle weakness can increase fall risk and decrease mobility. If the upper extremities are involved, hand dexterity may be lost, impeding self-care activities. Anthracyclines are known for causing cardiac dysfunction, which can lead to decreased endurance and activity tolerance, especially if concomitant lung metastases are present. ${ }^{2}$

Radiation therapy is often used for STS, and rarely for bony sarcomas. This raises the risk of short-term adverse events, such as delayed wound healing and infection. Radiation can cause late effects, including progressive fibrosis which persists and worsens even years after treatment is completed. Treatment given near joints can cause contractures from fascial tightening. Obstructed lymphatics can predispose a limb to developing lymphedema. Radiation given near neural structures can cause progressive nerve damage which is difficult to treat. The insidious nature of these changes and longtime course over which they occur make radiation effects especially difficult to diagnose and manage. Early identification is important to mitigate long-term irreversible effects. Pentoxifylline and tocopherol have shown some utility in slowing or arresting the progression of radiation fibrosis, though large studies are lacking. ${ }^{49,50}$

\section{Rehabilitation}

Recognition of the importance of rehabilitation services for cancer patients has increased in recent years, and sarcoma survivors in particular benefit from rehabilitation care. This is multifactorial, including the fact that many symptoms following sarcoma treatment involve the neuromusculoskeletal system and require the expertise of a physiatrist and multidisciplinary team. To underscore this, Stevenson et al found that patient function, regardless of the type of surgical treatment for limb sarcomas, was 
the most important contributor to quality of life. ${ }^{20}$ Rehabilitation is essential to optimize function in sarcoma patients. Members of a multidisciplinary rehabilitation team may include a physiatrist, nurse, physical therapist, occupational therapist, speech and language pathologist, orthotist, prosthetist, social worker, psychologist, rehabilitation engineer, and nutritionist. ${ }^{51}$ Though an individual may not need all of these services, they should be made available where cancer care is given.

Rehabilitation given before cancer treatment is undertaken, known as prehabilitation, can help increase tolerance for toxic and deleterious side effects. ${ }^{52}$ And while prehabilitation can potentially improve tolerance to chemotherapy, a key component of rehabilitation prior to treatment is patient counseling. If a choice between limb salvage and amputation must be made, patients should be fully educated on the advantages and disadvantages of each procedure including potential functional deficits.

Immediately after surgery, patients may benefit from inpatient rehabilitation. Patients who undergo amputation for sarcoma have been shown to make significant gains during inpatient rehabilitation compared to dysvascular controls, with a majority being discharged home. ${ }^{2}$ Similarly, patients who undergo limb salvage may require inpatient rehabilitation, especially in the setting of significant nerve damage or other post-operative complications. The goals of rehabilitation in this stage are primarily to strengthen the patient and provide adaptive equipment and strategies for functional deficits, all with the goal of safe discharge home. Considerations such as home set up, available assistance from friends and family members, patient employment, and others should be considered when tailoring a rehabilitation plan for each patient.

Patients continue to benefit from rehabilitation services in the outpatient setting. Those who undergo amputation require prosthetic evaluation and prescription after sufficient wound healing has occurred. The choice of prosthetic device is complex and depends on a number of factors, including patient age and comorbidities, prior functional level, and functional goals. These evaluations are best made by a prosthetist and physiatrist together. Long-term follow up is needed to monitor for wound complications and adjustments needed to prosthetic devices. Those who undergo limb salvage can benefit from rehabilitation follow up for pain management and orthoses to compensate from weakness due to nerve injury or neuropathy. Electrodiagnostic testing may also be helpful to determine the extent and potential for recovery from nerve damage. Those with radiation fibrosis should be provided with a home exercise program for stretching and be evaluated for stretching splints and orthoses. Patients with lung metastases may benefit from pulmonary rehabilitation as well.

Assisting patients with return to school and work is also vital to restoring QOL after sarcoma treatment. Returning to normal activities can improve sense of wellbeing. ${ }^{53}$ For children and adolescents, individualized education plans may be necessary in the short term upon returning to school. For adults, perceptions of employer accommodations are particularly important, and physicians play a crucial role in providing guidance for any work place alterations that are necessary. ${ }^{54}$ The patient's expected work duties should be enumerated and recommendations for activities that can and cannot be performed should be provided. Restrictions may be necessary on sport and work activities (e.g. avoiding high impact activities to protect endoprostheses). Any return to school or work after significant cancer treatment should be gradual and flexible, allowing time for the patient to readjust to their normal routine.

A mental health provider experienced with functional loss and chronic illness is an invaluable member of the interdisciplinary team for many oncology patients. The diagnosis and treatment of cancer can be a traumatic experience which patients handle differently, and patients treated for soft tissue sarcomas are at increased risk of developing anxiety, depression, and adjustment disorder related to grief from limb loss. ${ }^{55}$ This is true for all stages of the disease, including survivorship. ${ }^{56}$ Furthermore, sarcoma survivors are at increased risk of suicide compared to people without a history of sarcoma. ${ }^{57}$

Survivors should be screened for symptoms of depression. Psychosocial support has been shown to reduce depression and have a positive impact on patients, reinforcing the need for vigilance on the part of healthcare providers in monitoring the mental health of sarcoma patients and survivors. ${ }^{58}$ Antidepressant and anxiolytic medications may be helpful, even for those without premorbid mental health conditions.

\section{Lymphedema}

Lymphedema is an important diagnosis to manage when attempting to restore function with a multimodal rehabilitation program. Patients are at high risk of lymphedema in extremities affected by sarcoma. Lymph node removal, altered anatomy, weakness, and lymphatic obstruction by mass occupying lesions and radiation fibrosis all increase 
the risk of lymphedema before and after treatment. Identification of the cause will help guide treatment. The importance of early identification and treatment of lymphedema cannot be understated. If left untreated irreversible fatty infiltration can occur, leading to life-long limb volume increase. In rare and severe cases, lymphangiosarcoma can develop as well, underscoring rehabilitation's role in preventing secondary malignancy.

Infection is a common side effect of lymphedema. Proper drainage of lymph is necessary to mobilize the immune system against potential threats, and stagnation of this fluid provides a favorable environment for bacteria growth. Even minor cuts, abrasions, or insect bites can result in catastrophic infections. Any symptom of local infection warrants empiric treatment with antibiotics, usually directed against common skin flora. A thorough examination and laboratory work up should be performed to rule out abscess and deeper infection that may require drainage or surgical intervention.

The management of lymphedema usually begins with referral for lymphedema therapist. A physical or occupational therapist with specialized training in lymphedema management will evaluate the patient and provide treatment. Initial modalities consist of manual lymphatic drainage, where fluid is massaged from the limb, and compression bandaging to keep volume down after it is drained. When limb volume is stable, patients can be fitted for compression garments for long-term control. Patient and caregiver education are a vital part of this process. Those who are able should be instructed in performing manual lymph drainage at home. The warning signs of infection should be thoroughly reviewed and stressed to patients.

\section{Pain}

Adequate pain control is tantamount to maintaining function and QOL after sarcoma treatment. Sarcoma patients are at risk for many kinds of pain, and proper history and evaluation are important to ascertain the cause so appropriate management can be undertaken.

Neuropathic pain is common and often multifactorial. Nerve injury during limb salvage, phantom pain after amputation, and chemotherapy-induced peripheral neuropathy are possible cancer-related causes, which can be superimposed on other comorbidities such as diabetes and alcohol use. First-line pharmacologic management includes nervestabilizing agents such as gabapentin and pregabalin. Antidepressants such as serotonin norepinephrine reuptake inhibitors (e.g. duloxetine) and tricyclic antidepressants (e.g. nortriptyline) are also commonly utilized. Some antiepileptic medications such as carbamazepine can also be used but require more intensive monitoring for systemic side effects such as blood dyscrasias. Opiates should not be considered as first-line treatment for chronic pain control in patients with no evidence of disease. The medication(s) used should be titrated to therapeutic effect and tolerance of side effects. In addition to oral pharmacologic therapy, topical agents such as lidocaine preparations can also be effective. Desensitization modalities can be helpful for decreasing symptoms. Phantom limb pain can benefit from specialized modalities such as mirror therapy. Lidocaine injection of neuromas in severed nerves and proximal nerve blocks can also be beneficial.

Patients are also at risk for musculoskeletal pain due to altered biomechanics after surgery. Both muscle weakness in the area of limb salvage and prosthetic use after amputation can cause significant changes in the way patients move which can affect joints not originally affected by the sarcoma. For example, a patient who undergoes limb salvage for a distal femur osteosarcoma and has resultant quadriceps weakness can experience hip, sacroiliac, and lumbar spine dysfunction and pain years after a surgery as the cumulative effects of an altered gait lead to osteoarthritis and myofascial dysfunction. Physical and occupational therapy are important to strengthen weakened muscles and teach patients how to compensate for deficiencies. Orthotics to help enhance muscle function can be useful as well. A patient with weak quadriceps, for instance, could benefit from an ankle foot orthosis with an anterior shell to support knee extension.

\section{Measurement Tools}

A variety of measurement tools are available for sarcoma and cancer patients to assess function and QOL, which is important when gauging response to a rehabilitation program. The two most used specifically for sarcoma are the Musculoskeletal Tumor Society (MSTS) rating scale and the Toronto Extremity Salvage Score (TESS). The MSTS, formerly known as the Enneking score, is an evaluation of pain, function, and emotional acceptance with gait and ambulation items for lower extremity sarcomas and hand strength and dexterity items for upper extremity sarcomas which is completed by a medical provider. ${ }^{59,60}$ Conversely, the TESS is a patient-reported measure of 29 (upper extremity) or 30 (lower extremity) items focusing on physical functioning. ${ }^{60,61}$ Both are validated and used primarily for patients who have undergone limb salvage. In a study 
comparing the two, patient self-assessment (TESS) showed better perceived function than that ascertained by a physician's examination (MSTS) ${ }^{62}$ Both are recommended for routine evaluation of patients who have undergone limb salvage surgery.

The Karnofsky Performance Status (KPS) and Eastern Cooperative Oncology Group (ECOG) scores are estimations of global physical function widely used for oncology patients. ${ }^{51}$ Both focus primarily on function to the exclusion of QOL, and are ordinal scales that do not accurately reflect improvement of or decline in function as a patient's status changes. ${ }^{63}$ They are primarily used as global assessments of patient well-being to determine if patients are eligible for further treatment and as outcome measures for research purposes. Functional Independence Measures (FIMs) are used throughout rehabilitation to measure patient's progress toward independence. More recently, the World Health Organization (WHO) developed the International Classification of Function (ICF), a biopsychosocial model which takes a more inclusive view of function, emphasizing the underlying effect of disease on how patients interact with their environments. ${ }^{64}$

As opposed to physical functioning, QOL is a multifaceted concept which encompasses the effect disease has on patient's physical well-being, independence, relationships, environment, and spirituality. ${ }^{65}$ Increasing attention is being paid to quantifying and tracking QOL for cancer patients as life expectancy and treatment has improved. The 36-Item Short Form Survey (SF-36), Functional Assessment of Chronic Illness Therapy (FACIT) questionnaires, and Patient-Reported Outcomes Measurement Information System (PROMIS) surveys are widely used measures of QOL that are appropriate for this population.

\section{Conclusion}

Sarcomas are rare bone and soft tissue tumors usually treated with surgical excision, often with adjuvant chemotherapy and/or radiation. These tumors and their treatment can lead to multifaceted impairments in QOL and function which are best addressed with a multidisciplinary team. As survival improves with increased detection and improved treatments, increased attention has been focused on the long-term experience of survivors. Interdisciplinary collaboration is necessary to provide high quality, patient-centered care that fully addresses individual impairments and symptoms.

\section{Disclosure}

The authors report no conflicts of interest in this work.

\section{References}

1. Stiller C, Trama A, Serraino D, et al. Descriptive epidemiology of sarcomas in Europe: report from the RARECARE project. Eur $J$ Cancer. 2013;49(3):684-695. doi:10.1016/j.ejca.2012.09.011

2. Smith SR. Rehabilitation strategies and outcomes of the sarcoma patient. Phys Med Rehabil Clin N Am. 2017;28:171-180. doi:10.1016/j.pmr.2016.08.008

3. Custodio CM. Barriers to rehabilitation of patients with extremity sarcomas. J Surg Oncol. 2007;95:393-399. doi:10.1002/(ISSN)10969098

4. Gerrand C, Furtado S. Issues of survivorship and rehabilitation in soft tissue sarcoma. Clin Oncol. 2017;29:538-545. doi:10.1016/j. clon.2017.04.001

5. Singer S, Tap WD, Crago AM, et al. Soft tissue sarcoma. In: DeVita V, Lawrence T, Rosenberg S, editors. Cancer: Principles and Practice of Oncology. 10th ed. Wolters Kluwer Health; 2015:1253-1291.

6. Siegel G, Biermann JS, Chugh R, et al. The multidisciplinary management of bone and soft tissue sarcoma: an essential organizational framework. J Mulidiscip Healthc. 2015;8:109-115.

7. Smith SR, Reish AG, Andrews C. Cancer survivorship: a growing role for physiatric care. $P M$ R. 2015;7(5):527-531. doi:10.1016/j. pmrj.2014.12.004

8. Kwong TNK, Furtado S, Gerrand C. What do we know about survivorship after treatment for extremity sarcoma? A systematic review. Eur J Surg Oncol. 2014;40:1109-1124. doi:10.1016/j.ejso.2014.03.015

9. Eiser C, Cool P, Grimer RJ, et al. Quality of life in children following treatment for a malignant primary bone tumour around the knee. Sarcoma. 1997;1:39-45. doi:10.1080/13577149778461

10. Eiser C, Darlington AS, Stride CB, Grimer R. Quality of life implications as a consequence of surgery: limb salvage, primary and secondary amputation. Sarcoma. 2001;5:189-195. doi:10.1080/ 13577140120099173

11. Richardson A, Addington-Hall J, Amir Z, et al. Knowledge, ignorance and priorities for research in key areas of cancer survivorship: findings from a scoping review. Br J Cancer. 2011;105(Suppl 1): S82-S94. doi:10.1038/bjc.2011.425

12. Simon MA, Enneking WF. The management of soft-tissue sarcomas of the extremities. J Bone Joint Surg. 1976;58-A(3):317-327. doi:10.2106/00004623-197658030-00005

13. Ryndholm A, Rooser B. Surgical margins for soft-tissue sarcoma. J Bone Joint Surg. 1987;69-A(7):1074-1078. doi:10.2106/00004623198769070-00017

14. Chao AH, Mayerson JL, Chandawarkar R, Scharscmidt TJ. Surgical management of soft tissue sarcomas: extremity sarcomas. J Surg Oncol. 2015;111:540-545. doi:10.1002/jso.23810

15. Messerschmitt PJ, Garcia RM, Abdul-Karim FW, Greenfield EM, Getty PJ. Osteosarcoma. J Am Acad Orthop Surg. 2009;17:515-527. doi:10.5435/00124635-200908000-00005

16. Gilbert NF, Cannon CP, Lin PP, Lewis VO. Soft-tissue sarcoma. $J$ Am Acad Orthop Surg. 2009;17:40-47. doi:10.5435/00124635-20090100000006

17. DiCaprio MR, Friedlaender GE. Malignant bone tumors: limb sparing versus amputation. J Am Acad Orthop Surg. 2003;11:25-37. doi:10.5435/00124635-200301000-00005

18. Grimer RJ, Aydin BK, Wafa H, et al. Very long-term outcomes after endoprosthetic replacement for malignant tumours of bone. Bone Joint J. 2016;98-B(857-864). doi:10.1302/0301-620X.98B6.37417

19. Stevenson J, Tsagkozis P, Grimer R. Functional and quality of life outcomes in bone sarcoma following amputation, rotationplasty or limb-salvage. Expert Rev Qual Life Cancer Care. 2016;1 (4):303-312. doi:10.1080/23809000.2016.1203725

20. Houdek MT, Watts CD, Wyles CC, Rose PS, Taunton MJ, Sim FH. Functional and oncologic outcome of cemented endoprosthesis for malignant proximal femoral tumors. J Surg Oncol. 2016;114:501-506. doi:10.1002/jso.v114.4 
21. Haw CS, Gray DH. Excision arthroplasty of the hip. $J$ Bone Joint Surg. 1976;58-B(1):44-47. doi:10.1302/0301-620X.58B1.1270495

22. Gupta S, Kafchinski LA, Gundle KR, et al. Intercalary allograft augmented with intramedullary cement and plate fixation is a reliable solution after resection of a diaphyseal tumour. Bone Joint J. 2017;99-B:973-978. doi:10.1302/0301-620X.99B7.BJJ-2016-0996

23. Houdek MT, Wagner ER, Wilke BK, Wyles CC, Taunton MJ, Sim FH. Long term outcomes of cemented endoprosthetic reconstruction for periarticular tumors of the distal femur. Knee. 2016;23:167-172. doi:10.1016/j.knee.2015.08.010

24. Ng VY, Loui P, Punt S, Conrad EU. Allograft reconstruction for sarcomas of the tibia. Open Orthop J. 2017;11:189-194. doi:10.2174/1874325001711010189

25. Aksnes LH, Bauer HCF, Jebsen NL, et al. Limb-sparing surgery preserves more function than amputation. $J$ Bone Joint Surg. 2008;90-B:786-794. doi:10.1302/0301-620X.90B6.19805

26. Pardasaney PK, Sullivan PE, Portney LG, et al. Advantage of limb salvage over amputation for proximal lower extremity tumors. Clin Orthop Relat Res. 2006;443:201-208. doi:10.1097/01.blo.0000195413.16150.bc

27. Carty CP, Bennett MB, Dickinson IC, Steadman P. Electromyographic assessment of Gait function following limb salvage procedures for bone sarcoma. J Electromyogr Kinesiol. 2010;20(3):502-507. doi:10.1016/j. jelekin.2009.06.001

28. Carty CP, Dickinson IC, Watts MC, Crawford RW, Steadman P. Impairment and disability following limb salvage procedures for bone sarcoma. Knee. 2009;16(5):405-408. doi:10.1016/j.knee.2009.02.006

29. Furtado S, Grimer RJ, Cool P, et al. Physical functioning, pain and quality of life after amputation for musculoskeletal tumours. Bone Joint J. 2015;97-B:1284-1290. doi:10.1302/0301-620X.97B9.35192

30. Forni C, Gaudenzi N, Zoli M, et al. Living with rotationplasty: quality of life in rotationplasty patients from childhood to adulthood. J Surg Oncol. 2012;105(4):331-336. doi:10.1002/jso.22088

31. Wafa H, Reddy K, Grimer R, et al. Does total humeral endoprosthetic replacement provide reliable reconstruction with preservation of a useful extremity? Clin Orthop Relat Res. 2015;473:917-925. doi:10.1007/s11999-014-3635-5

32. Enneking WF, Dunham WK. Resection and reconstruction for primary neoplasms involving the innominate bone. J Bone Joint Surg. 1978;60-A(6):731-746. doi:10.2106/00004623-197860060-00002

33. Patterson FR, Peabody TD. Operative management of metastases to the pelvis and acetabulum. Orthop Clin North Am. 2000;31 (4):623-631. doi:10.1016/S0030-5898(05)70180-2

34. Mayerson JL, Wooldridge AN, Scharschmidt TJ. Pelvic resection: current concepts. J Am Acad Orthop Surg. 2014;22:214-222. doi:10.5435/JAAOS-22-04-214

35. Griesser MJ, Gillette B, Crist M, et al. Internal and external hemipelvectomy or flail hip in patients with sarcomas: quality-of-life and functional outcomes. Am J Phys Med Rehabil. 2012;91(1):24-32. doi:10.1097/PHM.0b013e318232885a

36. Azzarelli A, Quagliuolo V, Cerasoli S, et al. Chordoma: natural history and treatment results in 33 cases. $J$ Surg Oncol. 1988;37:185-191. doi:10.1002/(ISSN)1096-9098

37. Samson IR, Springfield DS, Suit HD, Mankin HJ. Operative treatment of sacrococcygeal chordoma. J Bone Joint Surg Am. 1993;75A:1476-1484. doi:10.2106/00004623-199310000-00008

38. Healey JH, Lane JM. Chordoma: a critical review of diagnosis and treatment. Orthop Clin North Am. 1989;20:417-426.

39. Chandawarkar RY. Sacrococcygeal chordoma: review of 50 consecutive patients. World J Surg. 1996;20:717-719. doi:10.1007/s002 689900110

40. Smith J, Ludwig RL, Marcove RC. Sacrococcygeal chordoma: a clinicoradiological study of 60 patients. Skeletal Radiol. 1987;16:37-44. doi:10.1007/BF00349926

41. Osler P, Bredella MA, Hess KA, et al. Sacral insufficiency fractures are common after high-dose radiation for sacral chordomas treated with or without surgery. Clin Orthop Relat Res. 2015;474:1-3.
42. Boriani S, Bandiera S, Biagini R, et al. Chordoma of the mobile spine: fifty years of experience. Spine (Phila Pa 1976). 2006;31:493-503. doi:10.1097/01.brs.0000200038.30869.27

43. Ruppert LM. Malignant spinal cord compression: adapting conventional rehabilitation approaches. Phys Med Rehabil Clin N Am. 2017;28:101-114. doi:10.1016/j.pmr.2016.08.007

44. Rao G, Suki D, Chakrabarti I, et al. Surgical management of primary and metastatic sarcoma of the mobile spine. J Neurosurg Spine. 2008;9:120-128. doi:10.3171/SPI/2008/9/8/120

45. Bergh P, Kindblom LG, Gunterberg B, Remotti F, Ryd W, MeisKindblom JM. Prognostic factors in chordoma of the sacrum and mobile spine: a study of 39 patients. Cancer. 2000;88:2122-2134. doi:10.1002/ (SICI)1097-0142(20000501)88:9<2122::AID-CNCR19>3.0.CO;2-1

46. Baratti D, Gronchi A, Pennacchioli E, et al. Chordoma: natural history and results in 28 patients treated at a single institution. Ann Surg Oncol. 2003;10:291-296. doi:10.1245/ASO.2003.06.002

47. Cheng EY, Ozerdemoglu RA, Transfeldt EE, Thompson RC. Lumbosacral chordoma. Prognostic factors and treatment. Spine (Phila Pa 1976). 1639-1645;1999:24.

48. Ho CH, Sung KC, Lim SW, et al. Chronic indwelling urinary catheter increase the risk of bladder cancer, even in patients without spinal cord injury. Medicine (Baltimore). 2015;94(43):e1736. doi:10.1097/ MD.0000000000001736

49. Williamson R, Kondziolka D, Kanaan $\mathrm{H}$, et al. Adverse radiation effects after radiosurgery may benefit from oral vitamin $\mathrm{E}$ and pentoxifylline therapy: a pilot study. Stereotact Funct Neurosurg. 2008;86:359-366. doi:10.1159/000163557

50. Pritchard J, Anand P, Broome J. Double-blind randomized phase II study of hyperbaric oxygen in patients with radiation-induced bracial plexopathy. Radiother Oncol. 2001;58:279-286. doi:10.1016/S01678140(00)00319-4

51. Tobias K, Gillis T. Rehabilitation of the sarcoma patient - enhancing the recovery and functioning of patients undergoing management for extremity soft tissue sarcomas. J Surg Oncol. 2015;111:615-621. doi: $10.1002 /$ jso.v111.5

52. Sliver JK, Baima J. Cancer prehabilitation: an opportunity to decrease treatment-related morbidity, increase cancer treatment options, and improve physical and psychological health outcomes. Am J Phys Med Rehabil. 2013;92:715-727. doi:10.1097/PHM.0b013e31829b4afe

53. van Muijen P, Duijts SFA, van der Beek AJ, Anema JR. Prognostic factors of work disability in sick-listed cancer survivors. $J$ Cancer Surviv. 2013;7:582-591. doi:10.1007/s11764-013-0297-3

54. Mehnert A, de Boer A, Feuerstein M. Employment challenges for cancer survivors. Cancer. 2013;119:2151-2159. doi:10.1002/cncr.28067

55. Trautmann F, Singer S, Schmitt J. Patients with soft tissue sarcoma comprise a higher probability of comorbidities than cancer-free individuals. A secondary data analysis. Eur J Cancer Care. 2017;26(6): e12605. doi:10.1111/ecc.12605

56. Paredes T, Canavarro MC, Simões MR. Anxiety and depression in sarcoma patients: emotional adjustment and its determinants in the different phases of disease. Eur J Oncol Nurs. 2011;15(1):73-79. doi:10.1016/j.ejon.2010.06.004

57. Siracuse BL, Gorgy G, Ruskin J, Beebe KS. What is the incidence of suicide in patients with bone and soft tissue cancer? Clin Orthop Relat Res. 2017;475(5):1439-1445. doi:10.1007/s11999-016-5171-y

58. Paredes TF, Canavarro MC, Simões MR. Social support and adjustment in patients with sarcoma: the moderator effect of the disease phase. J Psychosoc Oncol. 2012;30(4):402-425. doi:10.1080/ 07347332.2012 .684852

59. Enneking WF, Dunham W, Gebhardt MC, Malawar M, Pritchard DJ. A system for the functional evaluation of reconstructive procedures after surgical treatment of tumors of the musculoskeletal system. Clin Orthop Relat Res. 1993;286:241-246.

60. Parsons JA, Davis AM. Rehabilitation and quality-of-life issues in patients with extremity soft tissue sarcoma. Curr Treat Options Oncol. 2004;5:477-488. doi:10.1007/s11864-004-0036-0 
61. Davis AM, Bel RS, Badley EM, Yoshida K, Williams JI. Evaluating functional outcome in patients with lower extremity sarcoma. Clin Orthop Relat Res. 1999;358:90-100. doi:10.1097/00003086199901000-00012

62. Tunn PU, Pomraenke D, Goerling U, Hohenberger P. Functional outcome after endoprosthetic limb-salvage therapy of primary bone tumours - a comparative analysis using the MSTS score, the TESS and the RNL index. Int Orthop. 2008;32(5):619-625. doi:10.1007/ s00264-007-0388-8
63. Yasko AW, Reece GP, Gillis TA, Pollock RE. Limb-salvage strategies to optimize quality of life: the M.D. Anderson Cancer Center experience. $C A$ Cancer J Clin. 1997;47:226-238. doi:10.3322/canjclin.47.4.226

64. International Classification of Functioning, Disability and Health (ICF). Towards a Common Language for Functioning, Disability and Health. Geneva: World Health Organization; 2002:1-22.

65. WHO Programme on Mental Health. Measuring quality of life. Available from: http://www.who.int/mental_health/media/68.pdf. Accessed December 12, 2019.

\section{Publish your work in this journal}

Patient Related Outcome Measures is an international, peer-reviewed, open access journal focusing on treatment outcomes specifically relevant to patients. All aspects of patient care are addressed within the journal and practitioners from all disciplines are invited to submi their work as well as healthcare researchers and patient support groups.

Submit your manuscript here: http://www.dovepress.com/patient-related-outcome-measures-journal
The manuscript management system is completely online and includes a very quick and fair peer-review system. Visit http://www. dovepress.com/testimonials.php to read real quotes from published authors. 